\title{
Special Issue: Neuromuscular Cardiopulmonary Medicine in the Age of Emerging Therapies
}

\author{
David Birnkrant ${ }^{1}$ and Linda Cripe ${ }^{2}$ \\ ${ }^{1}$ MetroHealth Medical Center \\ ${ }^{2}$ Nationwide Children's Hospital
}

November 9, 2020

\begin{abstract}
Neuromuscular medicine is being revolutionized by new genetic and molecular therapies. The purpose of this Special Issue is to present an overview of these new therapies, to examine their cardiopulmonary effects, and to consider the future of neuromuscular cardiopulmonary care. The emphasis will be on Duchenne muscular dystrophy (DMD) and, to a lesser extent, spinal muscular atrophy (SMA), as these are the diseases with the most robust new drug development and related cardiopulmonary outcome data. This Special Issue contains articles on a number of relevant topics, including an overview of new genetic and molecular therapies for DMD, examining the currently available cardiopulmonary outcome data; and a critical examination of pulmonary outcome measures, assessing which outcomes should be used in treatment studies. We will provide an overview of cardiopulmonary phenotypic variability and discordance and their implications for assessing patient prognosis and response to therapies, and present a new perspective on neuromuscular-induced sleep-disordered breathing, viewed in the context of new and emerging therapies. Finally, we will consider which cardiac imaging modalities should be used as outcome measures in studies assessing DMD heart function, and take a look at novel therapeutic approaches to DMD heart disease, including management of rhythm disorders and heart failure, and the use of left ventricular assist devices.
\end{abstract}

Introduction to the Special Issue on Neuromuscular Cardiopulmonary Medicine in the Age of Emerging Therapies

David J. Birnkrant MD

Division of Pediatric Pulmonology and Sleep Medicine

Department of Pediatrics

MetroHealth Medical Center

Case Western Reserve University School of Medicine

Cleveland, Ohio

dbirnkrant@metrohealth.org

Linda H. Cripe MD

Division of Pediatric Cardiology

Nationwide Children's Hospital

The Ohio State University College of Medicine

Columbus, Ohio 
Linda.Cripe@nationwidechildrens.org

Correspondence to: David J. Birnkrant MD

Department of Pediatrics

2500 MetroHealth Drive

Cleveland, $\mathrm{OH} 44109$

dbirnkrant@metrohealth.org

Financial Disclosures: Dr. Birnkrant is a Clinical Advisor to Advanced Bio Machines PTE LTD (ABM Respiratory Care) and has a financial interest in patents licensed to ABM Respiratory Care. Dr. Cripe has no relevant financial disclosures.

Neuromuscular diseases often affect the respiratory muscles and, as a result, respiratory complications are a major cause of morbidity. Respiratory muscle weakness leads to hypoventilation and sleep-disordered breathing, as well as impaired coughing, which results in pneumonia, atelectasis, mucus plugging, aspirations and respiratory failure. ${ }^{1}$ The therapeutic focus has been on treating respiratory complications with assisted ventilation and assisted coughing. ${ }^{2}$ This approach has been successful in patients with Duchenne muscular dystrophy (DMD), extending patient survival into their 30's and beyond. ${ }^{3}$

With prolonged survival through anticipatory management of respiratory impairment, attention has shifted to neuromuscular cardiac complications. Neuromuscular diseases vary in the nature and severity of their cardiac involvement, with complications ranging from cardiomyopathy to conduction disorders and arrhythmias. In individual patients, the severity of cardiac muscle involvement does not necessarily correlate with the severity of respiratory (skeletal) muscle involvement, complicating assessment of baseline natural history and prognosis. ${ }^{4}$ DMD provides a good example of the rationale for the focus on cardiac therapies: when patients are managed with assisted ventilation, DMD cardiomyopathy becomes the primary cause of patient death, ${ }^{5}$ and so effective cardiac management strategies are needed.

Until recently, pharmacologic therapy for neuromuscular cardiopulmonary complications were quite limited. In DMD, glucocorticoids can improve long-term pulmonary function, allowing patients to achieve a higher peak FVC at a later age. While the therapy typically delays the onset of significant pulmonary impairment by a few years, it cannot arrest the progressive decline to very low levels of pulmonary function that typify the disease. ${ }^{6}$ With regard to DMD heart function, studies of glucocorticoid therapy have yielded mixed results, while certain conventional cardiac medications, such as angiotensin converting enzyme (ACE) inhibitors and aldosterone receptor antagonists, may slow short-term decline, with possible long-term benefits. ${ }^{7,8}$ However, DMD cardiomyopathy is progressive and while aggressive management of late-stage cardiac disease can be mitigating, in one study mean survival was only 8 months after the onset of congestive heart failure (CHF). ${ }^{9}$ Overall, existing pharmacologic options are very limited, and better therapies are badly needed, including cardiac therapies designed specifically for the particular cardiomyopathy associated with DMD.

Thus, it is a welcome development that neuromuscular medicine is being revolutionized by new genetic and molecular therapies. ${ }^{10,11}$ The purpose of this Special Issue is to present an overview of these new therapies, to examine their cardiopulmonary effects, and to consider the future of neuromuscular cardiopulmonary care. The emphasis will be on DMD and, to a lesser extent, spinal muscular atrophy (SMA), as these are the diseases with the most robust new drug development and related cardiopulmonary outcome data.

This Special Issue contains articles on a number of relevant topics, including an overview of new genetic and molecular therapies for DMD, examining the currently available cardiopulmonary outcome data; and a critical examination of pulmonary outcome measures, assessing which outcomes should be used in treatment studies. We will provide an overview of cardiopulmonary phenotypic variability and discordance and their implications for assessing patient prognosis and response to therapies, and present a new perspective on neuromuscular-induced sleep-disordered breathing, viewed in the context of new and emerging therapies. Finally, we will consider which cardiac imaging modalities should be used as outcome measures in studies 
assessing DMD heart function, and take a look at novel therapeutic approaches to DMD heart disease, including management of rhythm disorders and heart failure, and the use of left ventricular assist devices.

Our goal with this Special Issue is to explore topics at the interface of the new therapies, cardiopulmonary function, and patient survival. If we succeed, then this collection will raise more questions than it answers. For example, will the new therapies demonstrate long-term cardiopulmonary efficacy? What are the most clinically meaningful cardiopulmonary outcome measures, and what is the most valid methodology to assess them? What ethical issues will arise from the new therapies? For example, are the emerging therapies already viewed by clinicians, patients, families and other stakeholders as potential cures? If so, how has this perceptual shift transformed risk/benefit decisions regarding participation in new therapies and invasive treatments like intubation, implantable cardiac devices, and critical care? These issues affect all stakeholders: patients, their families, clinicians, patient advocates, and pharmaceutical and device developers. These issues also impact societal support of therapeutic advances, including the payors who fund patient care. This is the beginning of a new era in neuromuscular cardiopulmonary medicine, as it enters the age of translational medicine, and we hope that this Special Issue will introduce you to some of the key topics in this rapidly evolving field of medicine.

References

1. Birnkrant DJ, Bushby K, Bann CM et al. Diagnosis and management of Duchenne muscular dystrophy, part 2: respiratory, cardiac, bone health, and orthopaedic management. Lancet Neurol 2018; 17(4): 347-361.

2. Sheehan DW, Birnkrant DJ, Benditt JO, et al. Respiratory management of the patient with Duchenne muscular dystrophy. Pediatrics 2018; 142(Suppl 2): S62-S71.

3. Landfeldt E, Thompson R, Sejersen T, et al. Life expectancy at birth in Duchenne muscular dystrophy: a systematic review and meta-analysis.Eur J Epidemiol 2020; 35(7): 643-653.

4. Jin JB, Carter JC, Sheehan DW, Birnkrant DJ. Cardiopulmonary phenotypic discordance is common in Duchenne muscular dystrophy.Pediatr Pulmonol 2019; 54(2): 186-193.

5. Birnkrant DJ, Ararat E, Mhanna MJ. Cardiac phenotype determines survival in Duchenne muscular dystrophy. Pediatr Pulmonol 2016; 51(1): 70-76.

6. McDonald CM, Gordish-Dressman H, Henricson EK et al. Longitudinal pulmonary function testing outcome measures in Duchenne muscular dystrophy: Long-term natural history with and without glucocorticoids. Neuromuscul Disord 2018; 28(11): 897-909.

7. Raman SV, Hor KN, Woiciech M, Cardona A, et al. Stabilization of early Duchenne cardiomyopathy with aldosterone inhibition: results of the multicenter AIDMD trial. J Am Heart Assoc 2019; 8(19) doi.org/10.1161/JAHA.119.013501

8. Duboc D, Meune C, Lerebours G, et al. Effect of perindopril on the onset and progression of left ventricular dysfunction in Duchenne muscular dystrophy. J Am Coll Cardiol 2005; 45(6): 855-857.

9. Wang M, Birnkrant DJ, Super DM, Jacobs IB, Baher RC. Progressive left ventricular dysfunction and longterm outcomes in in patients with Duchenne muscular dystrophy receiving cardiopulmonary therapies. Open Heart 2018; 5(1):e000783. doi: 10.1136/openhrt-2018-000783.

10. Abreu NJ, Waldrop MA. Overview of gene therapy in spinal muscular atrophy and Duchenne muscular dystrophy. Pediatr Pulmonol 2020. doi.org/10.1002/ppul.25055

11. Maruyama R, Yokota T. Molecular diagnosis and novel therapies for neuromuscular diseases. J Pers Med 2020; 10(3): E129. doi: 10.3390/jpm10030129. 